\title{
Emission lines in the spectrum of the symbiotic star AG Draconis from 1997 to $2003^{\star, \star \star}$
}

\author{
L. Leedjärv ${ }^{1}$, M. Burmeister ${ }^{1,2}$, M. Mikołajewski ${ }^{3}$, A. Puss ${ }^{1,2}$, K. Annuk ${ }^{1}$, and C. Gałan ${ }^{3}$ \\ 1 Tartu Observatory, 61602, Tõravere, Estonia \\ 2 Department of Physics, University of Tartu, 4 Tähe Street, 50090, Tartu, Estonia \\ 3 Uniwersytet Mikołaja Kopernika, Centrum Astronomii, ul. Gagarina 11, 87-100, Toruń, Poland
}

Received 23 August 2003 / Accepted 9 October 2003

\begin{abstract}
The halo symbiotic star AG Dra has been monitored spectroscopically at the Tartu Observatory, Estonia, from September 1997 to March 2003. Altogether 189 spectra are used in the present paper. In addition, photometric UBVri observations at the Piwnice Observatory, Poland, from March 1996 to May 2003 are presented. Equivalent widths $(E W)$ of the strongest emission lines ( $\mathrm{H} \alpha, \mathrm{H} \beta, \mathrm{He}$ II $\lambda$ 4686, O VI Raman scattered $\lambda$ 6825) appear to be strongly variable, and in general, correlate with the $U$ brightness of the star. No apparent correlation with the orbital motion of the binary system was found, except for $\mathrm{H} \beta$ in the quiescent state. At the same time, there is a clear convergence of the high values of $E W$ to certain phases according to the shorter photometric period (378.5 days) proposed by Bastian (1998) and the pulsational period of the cool giant (355.27 days) by Gális et al. (1999). Higher resolution spectra show variability of the $\mathrm{H} \alpha$ profiles, with weak absorption component on the blue wing of the line around photometric minimum if the star is in quiescent state. The significant role of the cool giant in the activity of AG Dra is discussed.
\end{abstract}

Key words. stars: binaries: symbiotic - stars: winds, outflows - stars: individual: AG Dra

\section{Introduction}

By definition, symbiotic stars are stellar objects that simultaneously present a cool continuum and high excitation emission lines in their optical spectra. They are interpreted as interacting binaries consisting of a cool giant and a hot component, usually a white dwarf accreting matter from its companion. As a result of the mass-loss of the giant, symbiotic stars possess dense gaseous nebulae. Symbiotic stars undergo certain activity phases, often explained as due to episodes of thermonuclear burning of matter accreted by the white dwarf. These phases may last from tens of days to several years and recur on time scales of years, decades or centuries. During both quiescent and outburst states, the hot component ionizes gas surrounding the binary system to produce strong emission lines in a wide range of ionization states. A comprehensive summary of current knowledge about symbiotic stars can be found in Corradi et al. (2003).

$\mathrm{AG}$ Dra $\left(\mathrm{BD}+67^{\circ} 922\right)$ is an $\mathrm{S}$ (stellar) type symbiotic binary containing a $\mathrm{K}$ giant as a cool component. AG Dra

\footnotetext{
Send offprint requests to: L. Leedjärv, e-mail: leed@aai . ee

* Based on observations collected at Tartu Observatory, Estonia and Piwnice Observatory, Toruń, Poland

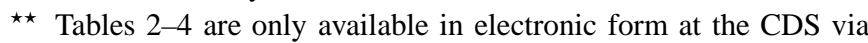
anonymous ftp to cdsarc.u-strasbg.fr $(130.79 .128 .5)$ or via http://cdsweb.u-strasbg.fr/cgi-bin/qcat?J/A+A/415/273
}

shows metal underabundance and its high systemic velocity of $-140 \mathrm{~km} \mathrm{~s}^{-1}$ (Schmid \& Nussbaumer 1993) or $-148 \mathrm{~km} \mathrm{~s}^{-1}$ (Schmid \& Schild 1997) as well as high Galactic latitude ( $b=41^{\circ}$ ) suggests that it belongs to the old halo population (Schmid \& Nussbaumer 1993).

The giant in AG Dra is hotter than in most symbiotic binaries and possibly brighter than luminosity class III (Friedjung 1997; Zhu et al. 1999). There are contradictions in spectral classification derived using different criteria, caused by metal underabundance. Mürset \& Schmid (1999) have adopted spectral class K2, while Zhu et al. (1999) restrict the possible range to K0-K3, luminosity class Ib or II. The range K4-K5 has been derived from the infrared colours (Kenyon 1988). The giant is enriched in heavy s-process elements (Smith et al. 1996). The surface temperature of the white dwarf companion has been estimated to be about $100000 \mathrm{~K}$ (Friedjung 1997) or $120000 \mathrm{~K}$ (Greiner et al. 1997; Schmid \& Schild 1997). AG Dra is also classified as one of the few Galactic supersoft X-ray sources (Greiner et al. 1997 and references therein; Ogley et al. 2002).

AG Dra is one of the most active symbiotic stars. Its light curve shows many active phases separated by quiescent periods. The most prominent active phases consisting usually of several outbursts start at about 14-15 year intervals, e.g. in $1936,1951,1966,1980,1994$. The mechanism of the outbursts is not yet clear. While many investigators adopt the scenario of thermonuclear events on the surface of the white 
dwarf, difficulties arise, in particular with time scales, when applying any more detailed model (cf. Gális et al. 1999; González-Riestra et al. 1999; Tomov \& Tomova 2002).

Any outburst of AG Dra is accompanied by changes in its continuous and line spectrum. Kafatos et al. (1993) and González-Riestra et al. (1999) have performed extensive studies of variability in the ultraviolet spectral region. There are many studies of optical spectra available, some of which we will refer later. Most recently, Tomov \& Tomova (2002) have published an analysis of hydrogen and helium emission lines during an active phase in 1996-1997. Our paper will deal with almost the same lines, and in addition with the Raman scattered $\mathrm{O}$ VI line at $\lambda 6825$, during the period from the decline of activity in September 1997 to quiescence in March 2003. In Sect. 2 we will describe the observations. Section 3 briefly describes the periodicities known in the AG Dra system. Section 4, devoted to the behaviour of the optical emission lines, is followed by a discussion and conclusions in Sect. 5 .

\section{Observations}

Our spectroscopic observations of AG Dra were carried out at the Tartu Observatory, Estonia, from September 1997 to March 2003. The $1.5 \mathrm{~m}$ telescope equipped with the Cassegrain grating spectrograph was used. Until March 1999, the spectra were registered with the SpectraSource Instruments CCD camera HPC-1 (Tek $1024 \times 1024$ chip, pixel size $24 \times 24 \mu \mathrm{m}$, Peltier cooled). Since March 1999 the spectra were obtained with the cryogenically (liquid nitrogen) cooled CCD camera Orbis-1 of the same company (Tek $512 \times 512$ chip, pixel size $24 \times 24 \mu \mathrm{m}$ ).

Altogether 189 spectra of AG Dra were obtained. The majority of the spectra have been recorded in two spectral regions which we call red and blue. 95 red spectra were taken with a linear dispersion of $28 \AA \mathrm{mm}^{-1}\left(\sim 0.66 \AA \mathrm{pix}^{-1}\right)$ at $\mathrm{H} \alpha$; they include emission lines of $\mathrm{H} \alpha$, He I $\lambda 6678$ and the Raman scattered $\mathrm{O}$ VI line at $\lambda 6825$. We have obtained also $22 \mathrm{H} \alpha$ spectra at higher dispersion ( $12 \AA \mathrm{mm}^{-1}$ or $\sim 0.26 \AA \mathrm{pix}^{-1}$ ). The overall wavelength range of both red and blue spectra depends on the CCD camera used. The new Orbis- 1 camera has half the columns of pixels than HPC-1 and thus the spectra obtained after March 1999 cover a shorter wavelength range. All the blue spectra include at least emission lines of $\mathrm{H} \beta$, He II $\lambda 4686$, and $\mathrm{He}$ I $\lambda$ 4713, those taken before March 1999 include also He I $\lambda 4471$ and $\mathrm{H} \gamma$. The linear dispersion of the blue spectra is about $36 \AA \mathrm{mm}^{-1}$ at $\mathrm{H} \beta\left(\sim 0.86 \AA \mathrm{pix}^{-1}\right)$. Typical exposure times were $5-15$ min for the (lower-dispersion) red spectra and 25-35 min for the blue spectra.

The spectra were reduced using the software package MIDAS provided by ESO. After standard procedures of subtracting the bias and sky background (and dark frame for the HPC-1 spectra) etc., the spectra were reduced to the wavelength scale using the $\mathrm{Ne}-\mathrm{Ar}$ (until October 1999) or $\mathrm{Th}-\mathrm{Ar}$ (after October 1999) hollow cathode lamp spectrum for calibration. The wavelength-calibrated spectra were normalized to the continuum, and positions, peak intensities and equivalent widths $(E W)$ of the emission lines were measured. In the case of some earlier spectra another software package, KASPEK, developed by K. Annuk at Tartu Observatory, was used for final steps of the reductions and to derive the above quantities for the spectral lines. Test reductions show that there are no systematic differences between the results obtained with these two packages.

We made some attempts to calibrate the measured $E W$ s of the emission lines into the absolute fluxes, using the published $U B V R$ photometry and our own observations. Interpolating broad-band fluxes and accounting for interstellar extinction with $E(B-V)=0.06$ (Mikołajewska et al. 1995; Viotti et al. 1983), we calculated continuum fluxes at the centres of the lines and thus also absolute fluxes emitted in the lines. Further analysis has shown that qualitatively the pattern of variability and conclusions derived are the same for both equivalent widths and absolute fluxes. In what follows, we have used equivalent widths as directly measurable quantities. The measured $E W$ s range from about $5 \AA$ to $140 \AA$; we have estimated their relative accuracy to be about $3-10 \%$.

Photoelectric $U B V r i$ photometry of AG Dra was carried out at Piwnice Observatory of the Nicolaus Copernicus University, Torun, Poland. The $60 \mathrm{~cm}$ Cassegrain telescope equipped with a one-channel photometer and EMI9558B photomultiplier tube working at ambient temperature was used for previous decade. Our instrumental performance of the $U B V$ bands was very close to the original Johnson photometry; accounting for atmospheric and transformation into the international system was carried out in the standard way. Unfortunately, the mean effective wavelengths of our instrumental $r$ and $i$ bands were $6390 \AA$ and $7420 \AA$, respectively - significantly shorter than in Johnson, or even Cousins photometric system. From the observations of tens of Johnson standard stars during the decade, we found formal transformation formulae into the international $R I$ system as follows:

$\Delta R=\Delta r+0.37 \Delta(R-I)$
$\Delta(R-I)=1.40 \Delta(r-i)$.

We have chosen the $\mathrm{F} 2$ star $\mathrm{BD}+66^{\circ} 934$ and the $\mathrm{A} 0$ star $\mathrm{BD}+67^{\circ} 926=\mathrm{HD} 145528$ as standard stars. In most cases, $\mathrm{BD}+66^{\circ} 934$ was used as a comparison star, whereas $\mathrm{BD}+67^{\circ} 926$ was used as a check. Only five observations (mainly searching for flickering) were done using BD+67926 as a comparison. Brightness and colours of those two stars remained constant over the whole period of observations. The obtained mean magnitudes in the Johnson system are presented in Table 1.

In the case of AG Dra, the flux in the instrumental $r$ and $i$ bands is a variable combination of the radiation from the cool giant and from the hot component (and possibly also from the nebula) of the symbiotic system. Given also the prevalence of strong $\mathrm{H} \alpha$ emission close to the $r$ band centre, we decided not to transform the observed $r$ and $i$ data into the standard system. The obtained $U, B, V, \Delta r$, and $\Delta i$ data from March 1996 to September 1999 are presented in Table 2 (available electronically only) where Col. 1 gives the times of observations in JD, Cols. 2-4 list $U, B$, and $V$ magnitudes, respectively, and Cols. 5 and 6 list $\Delta r$ and $\Delta i$. Accuracy of photometry is somewhat varuable, depending on the brightness of AG Dra, and has been estimated to be (in stellar magnitudes): $U-0.017, B-0.009$, 
Table 1. UBVRI data for the comparison stars.

\begin{tabular}{llllllll}
\hline \hline star & name & $V$ & $U-B$ & $B-V$ & $R$ & $R-I$ & $\begin{array}{l}\text { number of } \\
\text { observations }\end{array}$ \\
\hline comp. & BD $+66^{\circ} 934$ & $9.320 \pm 0.013$ & $0.193 \pm 0.013$ & $0.647 \pm 0.004$ & $8.795 \pm 0.010$ & $0.400 \pm 0.020$ & 14 \\
check & BD $+67^{\circ} 926=$ & $8.978 \pm 0.002$ & $0.083 \pm 0.005$ & $0.140 \pm 0.003$ & $8.819 \pm 0.002$ & $0.097 \pm 0.004$ & 40 \\
& $=$ HD 145528 & & & & & & \\
\hline
\end{tabular}

$V-0.009, R-0.006, I-0.012$ near maximum brightness, and $U-0.061, B-0.028, V-0.012, R-0.008, I-0.017$ near minimum brightness.

Recently, the photometer attached to the $60 \mathrm{~cm}$ reflector has been modernized significantly. Using the C31034 photomultiplier tube cooled to about 40 degrees below the ambient air temperature, and a new set of filters results in a system very close to the Johnson-Cousins $U B V R_{\mathrm{C}} I_{\mathrm{C}}$ photometry. Preliminary data from October 2001 to May 2003 are presented in Table 3 (available electronically only, and which lists JD, $U, B, V$ magnitudes, $\Delta R_{\mathrm{C}}$, and $\Delta I_{\mathrm{C}}$ similarly to Table 2) and will also be analyzed in this paper together with the earlier data and with the photometric data collected from Greiner et al. (1997), Montagni et al. (1996), Petrík et al. (1998), Skopal (1998), Skopal et al. (2002), Tomov \& Tomova (2000), Tomova \& Tomov (1998).

\section{Orbital and pulsational periods in AG Dra}

One of the main aims of our paper is to find possible periodicities in the behaviour of emission lines in the spectrum of AG Dra. In this section we briefly review the known periods. Meinunger (1979) found from quiescent photometric data the $U$ brightness variability with a period of 554 days and an amplitude of about 1 magnitude. Minima of those variations occur at the lower conjunction of the cool component. This turned out to be the orbital period. Gális et al. (1999) proposed a revised value $549.73 \pm 1.59$ from radial velocities of the absorption lines. Fekel et al. (2000) investigated infrared absorption lines, and their result, $548.65 \pm 0.97$, coincides with that of Gális et al. within the error limits.

Claims for shorter periods exist. Bastian (1998) found a 378 d 5 photometric period from visual observations. Friedjung et al. (1998) detected somewhat shorter periods, 356 days in the $B$, and 350 days in the $V$ band, respectively. Gális et al. (1999) found a similar period, $355.27 \pm 1.82$, also in the radial velocities of the absorption lines, and suggested this to be due to the pulsations of the K giant. Friedjung et al. (2003) once more confirmed presence of the latter period. In the formulae presented below, we have adjusted the ephemerides given by the above authors, so that zero points of all the ephemerides are close to the start of the large outburst in 1994. Integers subtracted from $E$ show the number of epochs by which the original starting points are shifted. Equations (1)-(3) describe orbital ephemerides, and Eqs. (4)-(5) describe pulsational and activity cycles.

Meinunger

$\mathrm{JD}(U \max$, quiesc $)=2449426+554 \cdot(E-19)$
Gális et al.

$\mathrm{JD}(U \min$, quiesc $)=2449126.47+549.73 \cdot(E-10)$.

Shifting the zero point of the latter ephemeris to the maximum $U$ brightness would yield:

$\mathrm{JD}(U \max$, quiesc $)=2449401.33+549.73 \cdot(E-10.5)$

Bastian

$\mathrm{JD}(U \max$, active $)=2449524.2+378.5 \cdot(E-4)$

Gális et al.

$\mathrm{JD}\left(V_{\mathrm{r}} \min \right)=2449554.31+355.27 \cdot(E-4)$.

Gális et al. (1999) noted the that ratio of their orbital period to the pulsational one is very close to $14 / 9$. This arises an interesting possibility that resonances between the (non-radial?) pulsations of the cool giant and orbital motion might increase the accretion rate onto the white dwarf, and thus trigger its outburst activity. Outbursts are accompanied by changes in the continuous and line spectrum of AG Dra, but possible periodicities and phase dependencies of the emission lines in the spectrum of AG Dra have not been thoroughly investigated. For instance, Smith \& Bopp (1981) found changes in the profiles of the Balmer lines well correlated with the 554-day photometric period, with a blueward component being visible in the Balmer emissions at photometric minimum (phase $0.55 \mathrm{ac}-$ cording to their Fig. 2). Iijima et al. (1987) found intensities of the emission lines to be variable, not attempting to link the variability to the orbital motion. Kaler (1987) found that in the quiescent state the fluxes of $\mathrm{H} \alpha$ and $\mathrm{H} \beta$ follow the variations in the Strömgren $u$ band, but not in a simple manner. He also reported that variations in $\mathrm{H} \beta$ may be modulated by a semiperiodic, roughly yearly variation in the wind flow of the giant component, which could be related to its own intrinsic variability. This is the first claim of a possible period of about one year in the AG Dra system. In the case of the flux of the He II $\lambda 4686$ line, Kaler (1987) found mostly only sporadic variations.

\section{Analysis of spectral lines}

\subsection{Intensities and profiles of the emission lines}

The period covered by our spectroscopic observations includes the end of the last active phase of AG Dra, and the relatively quiet state following large outbursts. In Fig. 1 we present a long-term $U$ light curve compiled from the literature (see references at the end of Sect. 2), and from our own observations at Piwnice Observatory. Our spectroscopic observations start at 


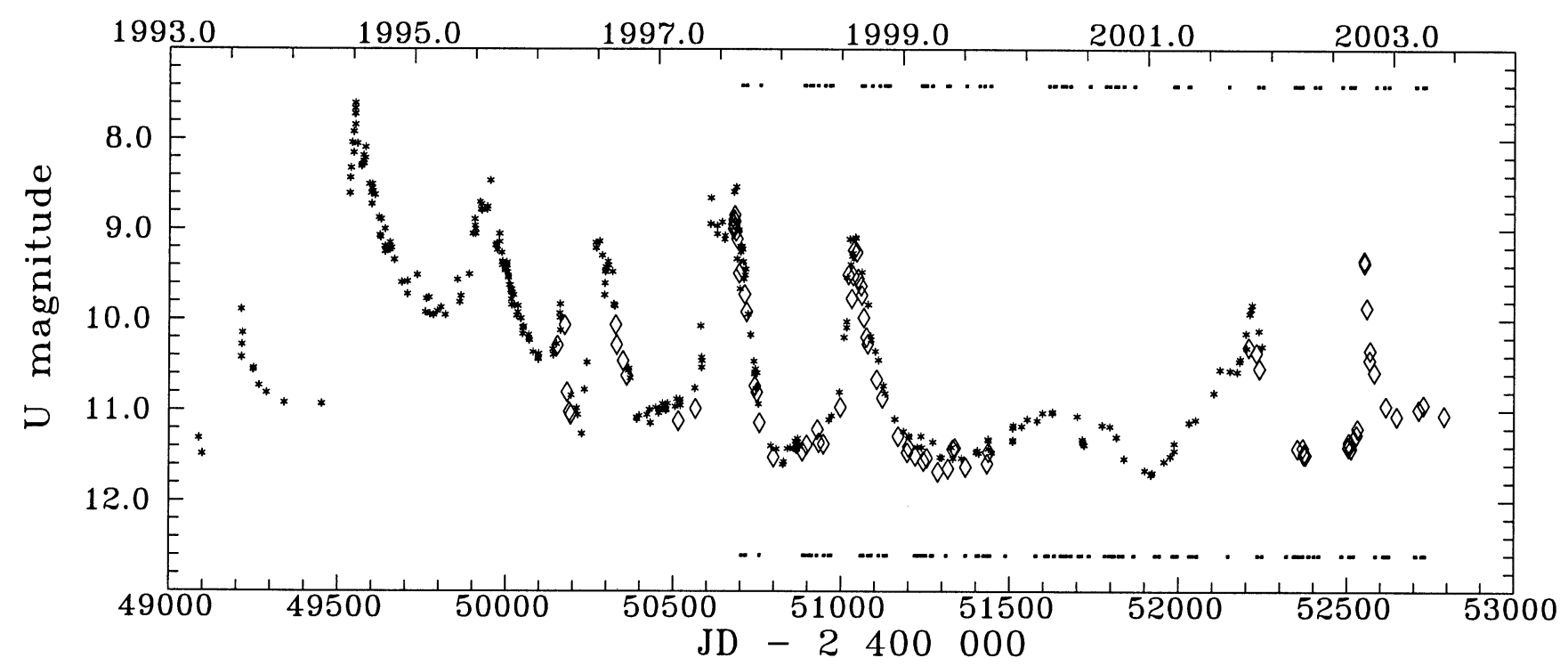

Fig. 1. Photoelectric $U$ light curve of AG Dra from 1993 to 2001. Our observations are shown with the open diamonds. Small dots at the upper $x$-axis denote the moments when our blue spectra were taken, and those at the lower $x$-axis the same for the red spectra.

JD 2450703 , at the decline from the light maximum in 1997, cover the next light maximum in 1998 (around JD 2451050 ) and the following quiescent phase which was interrupted by a moderate brightness rise after JD 2452 000. Particularly interesting is a short-time steep brightness rise around JD 2452550 (October 2002), but unfortunately this episode was so short that we did not succeed in obtaining spectra at the same time. The closest date of spectral observations was JD 2452586 , and there were no peculiarities detected in those spectra.

It is known from earlier spectroscopic studies that intensities of the emission lines in the spectrum of AG Dra can vary significantly (Iijima et al. 1987; Mikołajewska et al. 1995; Tomov \& Tomova 2002; Tomova \& Tomov 1999; Viotti et al. 1998). We are interested in the time scales of the variability, namely, are variations of the emission lines correlated with orbital motion, or with shorter periods of variability around 350-380 days, or are there no correlations at all. In Table 4 the following information is given: Col. 1 lists the moments of observations in JD, Cols. 2 to 5 list the $E W$ for the Raman scattered line $\lambda$ 6825, $\mathrm{H} \alpha, \mathrm{H} \beta$ and He II $\lambda$ 4686, respectively, and Cols. 6 to 9 give the phases computed from the ephemerides by Meinunger (1), Gális et al. (2), Bastian (4), and Gális et al. (5).

Time variability of the $E W \mathrm{~s}$, in general, follows the photometric behaviour of AG Dra. In Fig. 2 we present correlations of the logarithms of $E W \mathrm{~s}$ for some emission lines with the $U$ brightness. As can be seen, the logarithms of $E W s$ of He II $\lambda 4686$ and Raman scattered O VI $\lambda 6825$ follow rather well the linear approximation drawn through the data points. For Balmer lines the linear fit is not so good. In principle, we can discriminate two regions in Fig. 2. The area where $U \geq 11 \mathrm{mag}$ corresponds to the quiescent phase, while this with $U<11$ mag corresponds to the outbursts and brightenings like those in 1997, 1998, 2001 and 2002. EWs of the Balmer lines are variable during quiescence, the character of the variability being mostly random. One can notice about twofold differences in the $E W \mathrm{~s}$ at the same $U$ magnitude. The higher

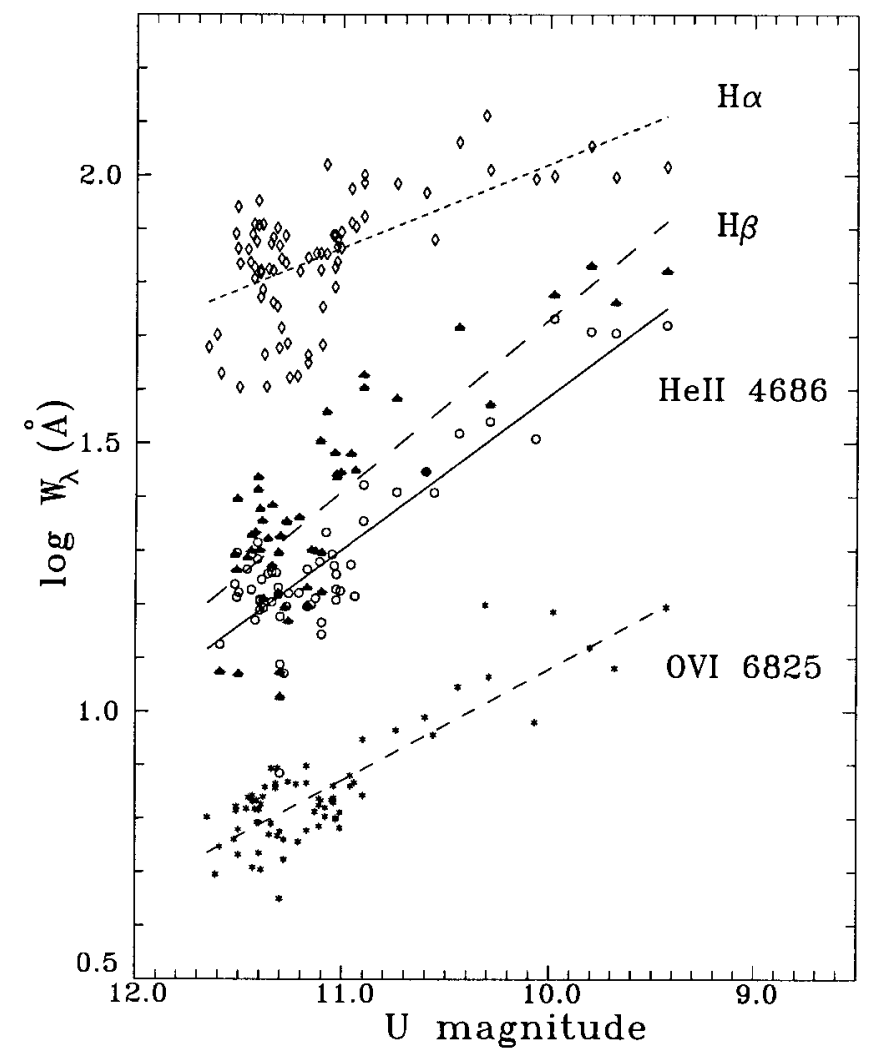

Fig. 2. Equivalent widths of the emission lines depending on the $U$ brightness. Filled triangles denote $\mathrm{H} \beta$ and open circles He II $\lambda 4686$.

excitation lines (He II $\lambda 4686$ and O VI $\lambda$ 6825) are somewhat less variable during quiescence. In bright phases $(U<11 \mathrm{mag})$ the $E W$ s of the lines grow linearly with the $U$ brightness, but for Balmer lines there is a bigger scatter. The neutral helium lines are much weaker than other lines considered here, we have not included their analysis in the present paper. 


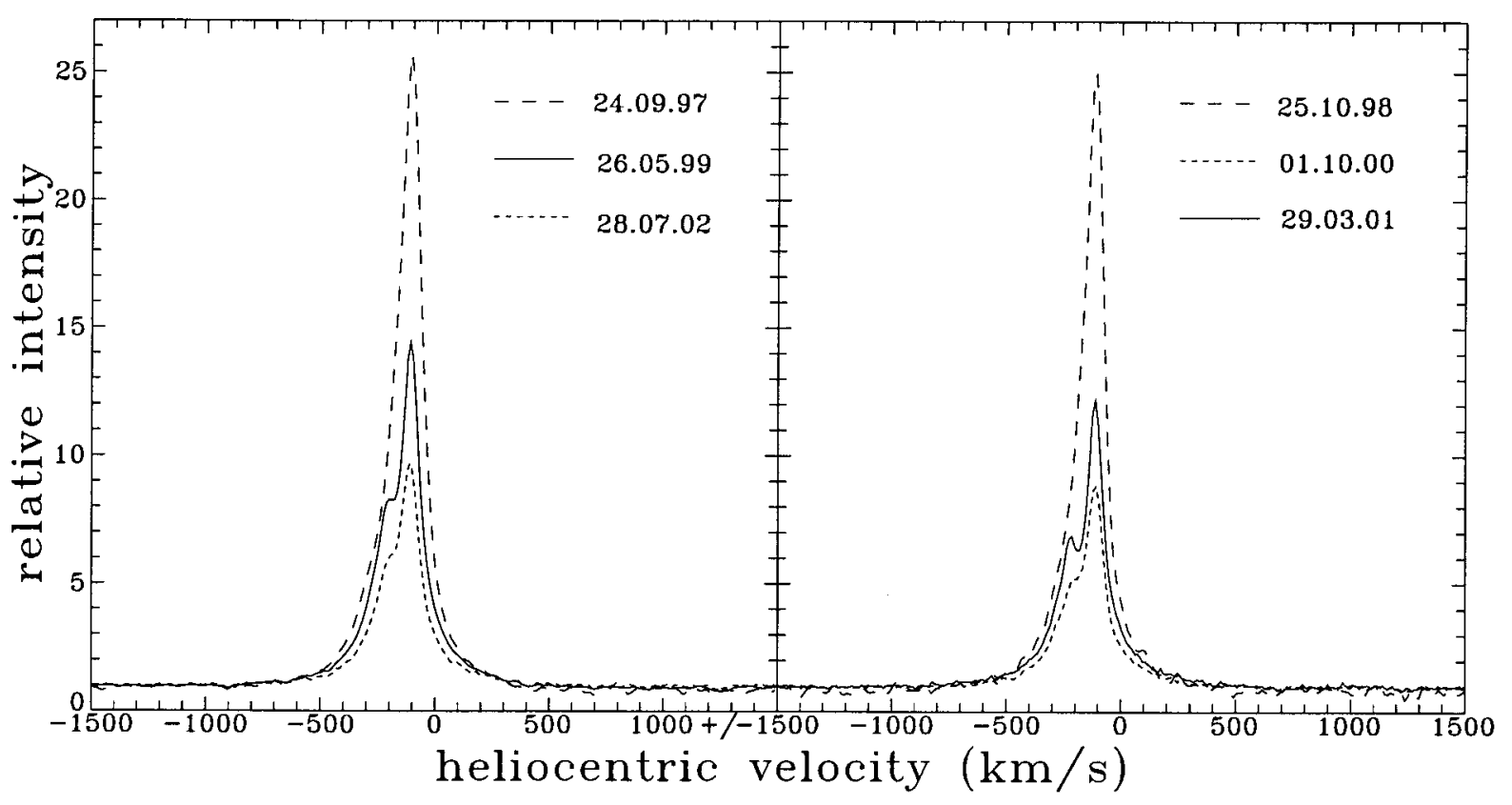

Fig. 3. Some examples of the $\mathrm{H} \alpha$ profiles of AG Dra.

Many authors have reported that the $\mathrm{H} \alpha$ profiles of AG Dra are single-peaked emission lines with a shoulder on the blue wing at all phases (e.g. Tomov \& Tomova 2002; Tomova \& Tomov 1999 and references therein). In addition, extended symmetric low intensity wings reaching the continuum level at about $\pm(1500-2000)$ or even $3000 \mathrm{~km} \mathrm{~s}^{-1}$ have been observed frequently. Robinson et al. (1994) claimed detecting a double-peaked $\mathrm{H} \alpha$ profile on May 4, 1990. However, closer inspection of their Fig. 6 allows us to take this claim with some reservation. Our spectra with higher dispersion $\left(12 \AA \mathrm{mm}^{-1}\right)$ in the $\mathrm{H} \alpha$ region have revealed the $\mathrm{H} \alpha$ profile to be singlepeaked, with asymmetry on the blue side at all times. Some of the profiles are rather smooth, while sometimes there has been quite a remarkable dip on the blue side of the line, which seems to be due to self-absorption. This dip is most pronounced on March 29, 2001 (Fig. 3), and this spectrum very much resembles that from May 4, 1990 in the paper by Robinson et al. (1994). The deepest absorption is placed at about $-185 \mathrm{~km} \mathrm{~s}^{-1}$ (or $-37 \mathrm{~km} \mathrm{~s}^{-1}$ from the line centre, if we take into account the systemic velocity $-148 \mathrm{~km} \mathrm{~s}^{-1}$ ). If we consider the peak blueward of this absorption as an emission peak, its radial velocity is about $-220 \mathrm{~km} \mathrm{~s}^{-1}$. In all cases, when some (absorption) structure is seen in the blue wing of the $\mathrm{H} \alpha$ line, it is placed between $-220 \mathrm{~km} \mathrm{~s}^{-1}$ and $-170 \mathrm{~km} \mathrm{~s}^{-1}$ (or about -70 to $-20 \mathrm{~km} \mathrm{~s}^{-1}$ from the line centre).

We pay attention to the $\mathrm{H} \alpha$ profiles from May 26, 1999 and October 1, 2000 where a plateau-like feature can be noticed on the blue side (between the velocities of about -215 to -170 and -220 to $-185 \mathrm{~km} \mathrm{~s}^{-1}$, respectively). The general tendency is that absorption features in the blue wing of $\mathrm{H} \alpha$ are more pronounced, when the peak intensity and $E W$ of the line are low. Profiles of the stronger lines are smoother.

Eight of our 22 higher-dispersion $\mathrm{H} \alpha$ profiles show this kind of absorption features. These occasions fall between phases 0.304 and 0.670 according to the photometric ephemeris by Meinunger (Eq. (1)). Thus, such features are observed around photometric minimum only. According to the photometric ephemeris by Bastian (Eq. (4)) all but one of the "structured profiles" occur between the phases 0.504 and 0.821 , but the profile with the rather remarkable plateau on October 1 , 2000 appears to be present at $\Phi_{\text {Bastian }}=0.063$. Finally, the pulsational ephemeris from Gális et al. (1999) (Eq. (5)) tells us that most of the "structured profiles" appear between phases 0.767 and 0.985, however, there are two exceptions: October 1, 2000 $\left(\Phi_{\text {puls }}=0.375\right)$ and July $28,2002\left(\Phi_{\text {puls }}=0.248\right)$.

The possible double-peaked emission profile observed by Robinson et al. (1994) on May 4, 1990, corresponds to $\Phi_{\text {Meinunger }}=0.455, \Phi_{\text {Bastian }}=0.016$ and $\Phi_{\text {puls }}=0.671$. Viotti et al. (1998) also report possible indication of a double-peaked structure in $\mathrm{H} \alpha$ in May 1992. They have not specified the date, but fixing it as May 16, 1992 yields $\Phi_{\text {Meinunger }}=0.796 \pm$ $0.027, \Phi_{\text {Bastian }}=0.979 \pm 0.040, \Phi_{\text {puls }}=0.762 \pm 0.042$. The $\mathrm{H} \alpha$ profile from January 16, 1995 in Viotti et al. (1998) can also be considered as a "structured" one; this corresponds to the phases $\Phi_{\text {Meinunger }}=0.556, \Phi_{\text {Bastian }}=0.554$ and $\Phi_{\text {puls }}=0.506$.

Phase coverage of our 22 high-dispersion spectra is quite poor and not uniform. Lower-dispersion spectra, unfortunately, have a resolution not sufficient to follow the profile variations exactly. However, taking into account also the above cited information from the literature, we may state, with some reservation, that absorption structures in the $\mathrm{H} \alpha$ profile of AG Dra mostly appear around the photometric minimum of the approximately 550-day orbital period. As to possible pulsational periods, those features also appear in certain phase ranges, but with some exceptions. At the same time, most of the $\mathrm{H} \alpha$ profiles from 1997 and 1998, when the star was in an outburst state, are rather smooth, independently of phase. 

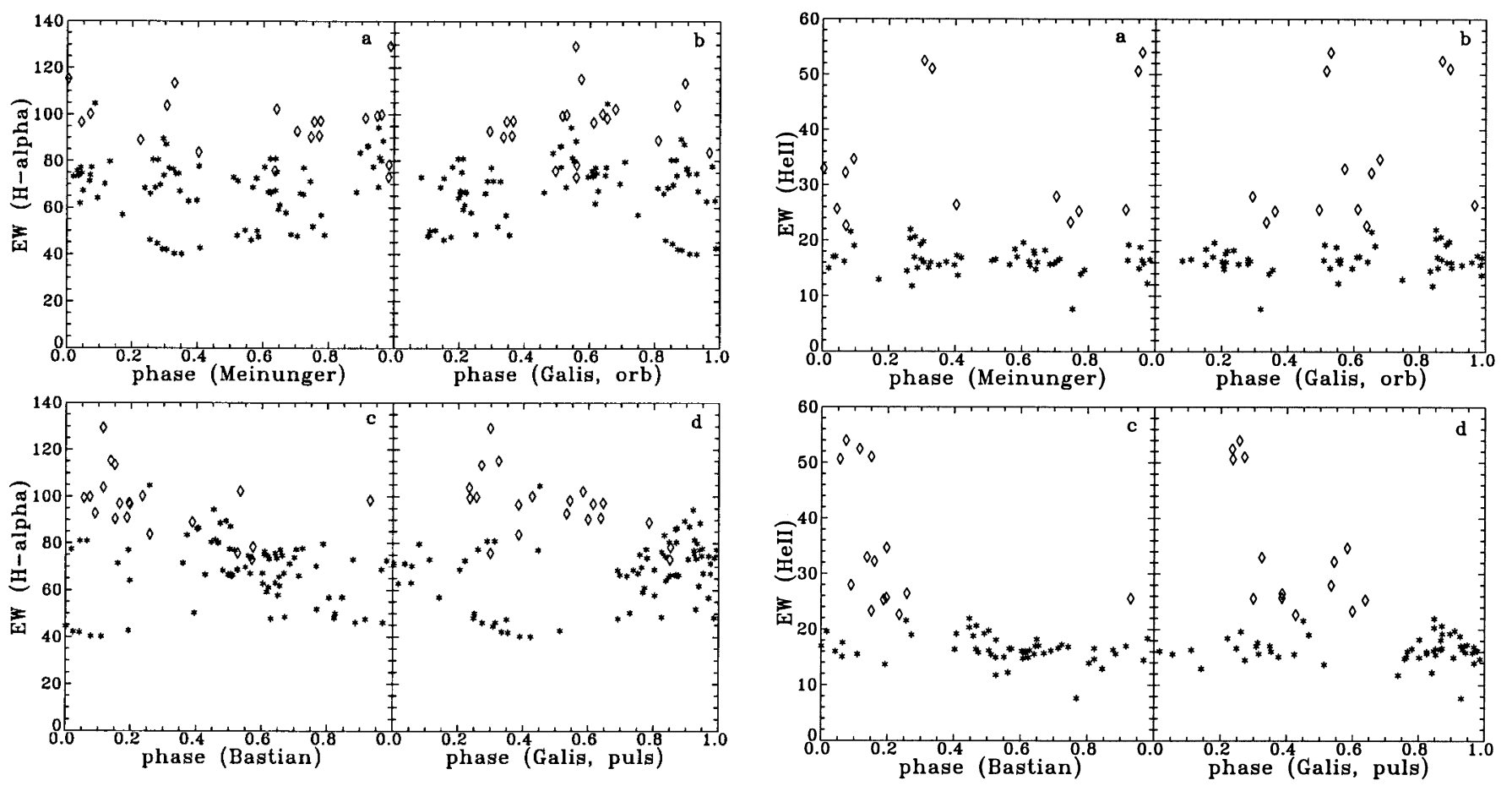

Fig. 4. $E W$ of the $\mathrm{H} \alpha$ line depending on different phases. Asterisks correspond to the quiescence $(U \geq 11)$, open diamonds mark the states when $U<11$.

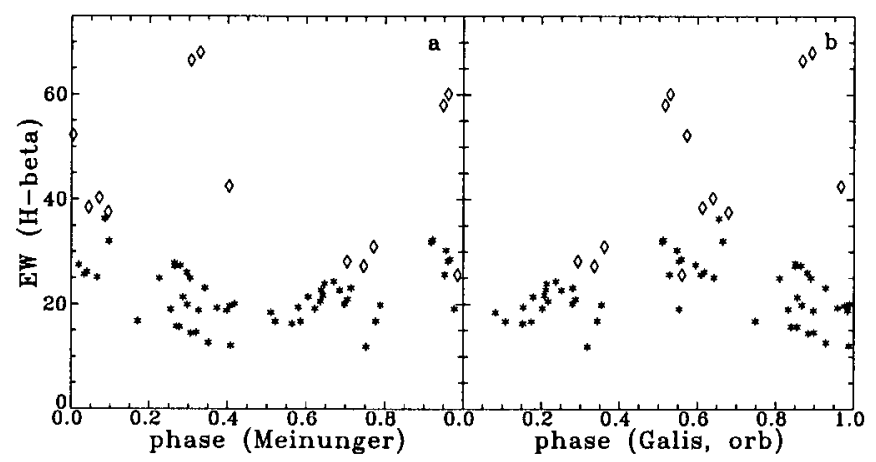

Fig. 6. The same as in Fig. 4 for He II $\lambda 4686$.
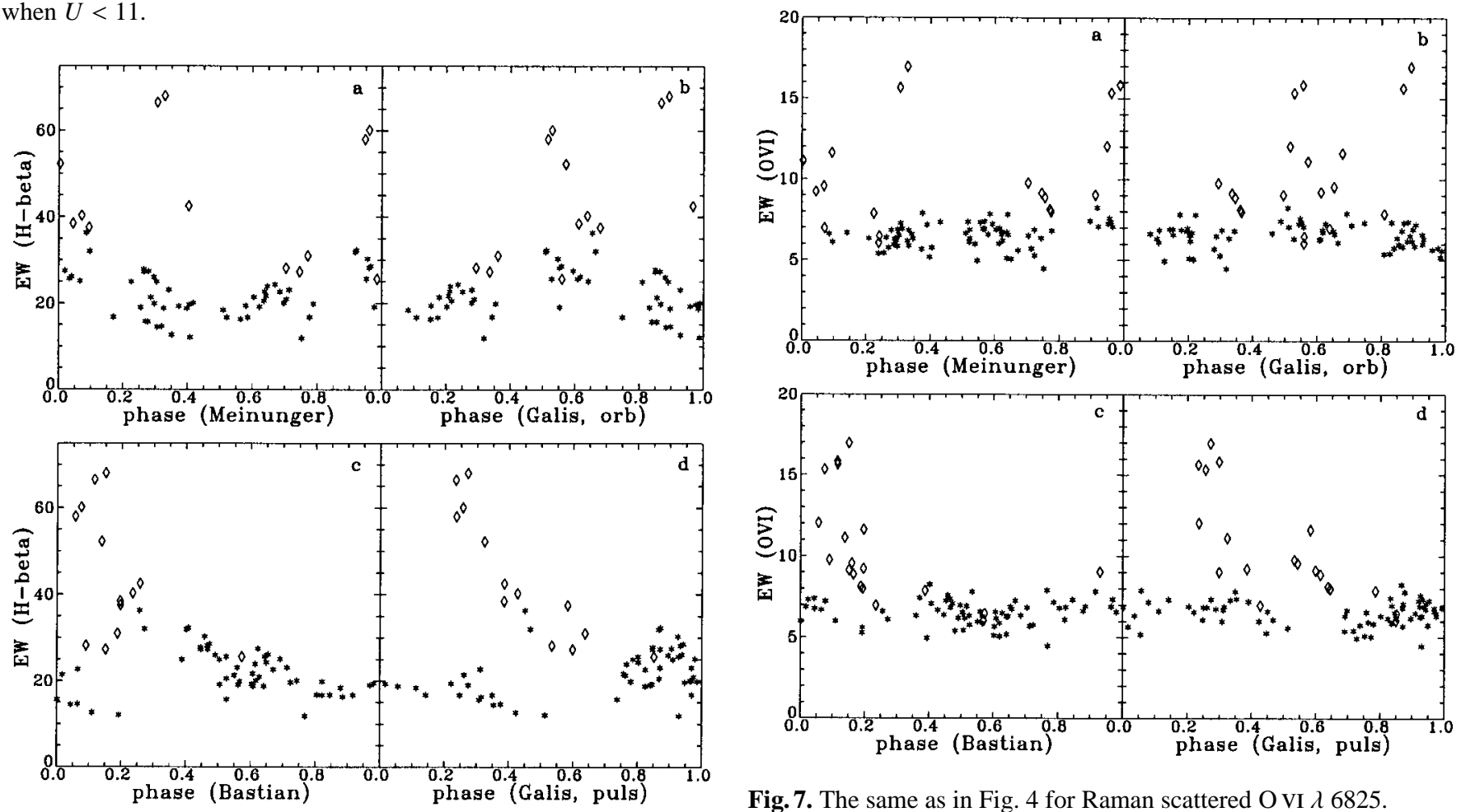

Fig. 5. The same as in Fig. 4 for $\mathrm{H} \beta$.

\subsection{Search for a period}

In this subsection, we will attempt to find which of the known ephemerides for AG Dra, if any, match the variations of the emission line intensities. Figures 4-7 present phase dependencies for $E W \mathrm{~s}$ of the lines $\mathrm{H} \alpha, \mathrm{H} \beta$, He II $\lambda 4686$ and Raman scattered O VI $\lambda 6825$, respectively. Each graph consists of four

Fig. 7. The same as in Fig. 4 for Raman scattered O vi $\lambda 6825$.

panels: two upper panels ( $a$ and $b$ ) show dependence on orbital phase (Eqs. (1) and (2), respectively). Two lower panels (c and d) represent possible pulsational phases of the cool giant (Eqs. (4) and (5)).

$E W \mathrm{~s}$ of the $\mathrm{H} \alpha$ line show the greatest scatter. It is hard to find regularities in any of the panels of Fig. 4. Closer inspection reveals some similarities to Figs. 5-7 but the general conclusion is that the lowest and highest values of $E W$ 
have been observed at practically the same phases by all the ephemerides. When applying more data from the literature, the picture becomes even more confused. For example, Viotti et al. (1998) measured the $E W$ of the $\mathrm{H} \alpha$ line as low as $19.1 \AA$ on December $27,1990\left(\Phi_{\text {Meinunger }}=0.883, \Phi_{\text {Bastian }}=\right.$ $\left.0.642, \Phi_{\text {Galis,puls }}=0.337, \Phi_{\text {Galis,orb }}=0.411\right)$. Tomova \& Tomov (1999) report the very high $E W$ value of $184 \AA$ on January 23, 1994 , at quite similar phases, when the star was still in quiescence $\left(\Phi_{\text {Meinunger }}=0.911, \Phi_{\text {Bastian }}=0.610, \Phi_{\text {Galis,puls }}=\right.$ $\left.0.500, \Phi_{\text {Galis,orb }}=0.455\right)$.

Other emission lines demonstrate more regular behaviour. One may notice a minimum in the quiescent time $E W$ s of $\mathrm{H} \beta$ around $\Phi_{\text {Meinunger }}=0.5$, but for other emission lines it is hard to find any conspicuous phase dependence of "quiescent" values. The "outburst" values (marked with diamonds) are distributed quite randomly in both orbital phases. But in pulsational phases, practically all those higher values of $E W \mathrm{~s}$ fall into a narrow range of phases: $\Phi_{\text {Bastian }} \approx 0.05 \ldots 0.25$ or $\Phi_{\text {Galis,puls }} \approx$ $0.20 \ldots 0.55$. Although the number of points is rather small, we consider such a convergence real. This is confirmed by the similarity of the patterns for all three lines (note, in particular, a very close similarity in the behaviour of the He II $\lambda 4686$ and the Raman $\lambda 6825$ lines).

All the cited periods apply to the photometric data or radial velocities of the absorption lines. We made an attempt to perform period analysis also for the $E W \mathrm{~s}$ of the emission lines. The software package ISDA (Irregularly Spaced Data Analysis) compiled by Dr. J. Pelt from Tartu Observatory was used (see http://wWw.aai.ee/ ${ }^{\sim}$ pelt/soft.htm\#ISDA). We searched for the minimum dispersion in the period range 100-1000 days using the least square method. For all four emission lines used ( $\mathrm{H} \alpha, \mathrm{H} \beta$, He II $\lambda 4686$ and Raman scattered $\lambda$ 6825) a global minimum at frequencies of about 0.0025-0.0027 day $^{-1}$ was found (corresponding formal periods range from 389.8 to 395.8 days). However, given the small number of data points and large scatter (especially for $\mathrm{H} \alpha$ ), one should not attribute too much confidence to the periods found this way. Being aware of caveats and risks, while interpreting periods close to one year, we leave open the question, whether there really is a period around 390 days in the intensities of the emission lines, and if yes, why it is different to both orbital and pulsational period. More observational data, especially in the outbursts, would be needed to solve this problem.

\section{Discussion and conclusions}

AG Dra is considered to be a classical symbiotic star. Its behaviour is summarized by González-Riestra et al. (1999) as follows: AG Dra is generally in a nearly permanent normal state. This state is occasionally subject to perturbations which give rise to a substantial increase of the optical luminosity (the outbursts). It is known from many studies that the most active phases (which may consist of several outbursts) of AG Dra repeat with about 13-15 year intervals. González-Riestra et al. (1999) have distinguished between cool and hot outbursts of AG Dra. The major active phases that started in 1980 and 1994 contain several cool outbursts during which the He II Zanstra temperature drops and the expanding white dwarf cools, while its radius increases by factors of 2 to 6 . In smaller-scale hot outbursts observed in 1985-1986, the He II Zanstra temperature increased. The decay time of those weaker outbursts was substantially shorter than in other active phases. X-ray flux from AG Dra drops significantly in both cool and hot outbursts (Greiner et al. 1997; González-Riestra et al. 1999). This is quite obvious for the cool outbursts, when the white dwarf is expanding and cooling. For hot outbursts it is assumed that the presence of an extended atmosphere and an opaque hot wind produce a high energy cutoff which would drastically reduce the soft X-ray flux.

There are at least two other supersoft X-ray sources for which alternating X-ray on- and off-states have been discovered, RX J0513.9-6951 (Pakull et al. 1993; Reinsch et al. 1996) and CAL 83 (Kahabka 1998; Greiner \& DiStefano 2002). The time scales, however, are much shorter than in AG Dra. For example, in RX J0513.9-6951 X-ray on-states of duration $\sim 30$ days occur during optical dips of similar duration, while X-ray off-states occur during optical high-states. The on/off pattern has a recurrence time of $\sim 100-200$ days (Southwell et al. 1996). A limit cycle has been proposed to explain the behaviour of RX J0513.9-6951 in which expansion of the white dwarf atmosphere (due to an increase in the mass-accretion rate) enhances the irradiation of the accretion disc and the mass-flow through the disc (Reinsch et al. 2000). Thus, the time scale involved is the viscous time of the disc.

There is no obvious evidence of an accretion disc in the AG Dra system. Rapid photometric variability on the time scales of minutes would be one almost certain indicator of an accretion disc. Sokoloski et al. (2001) in their comprehensive study of symbiotic stars failed to detect such a variability in AG Dra. Very broad wings of the Balmer and He II emission lines during the outbursts indicate the presence of a highvelocity stellar wind. Such a wind may be generated in an accretion disc or by a hot stellar object. Tomova \& Tomov (1999) and Tomov \& Tomova (2002) have argued that available observational data do not support the existence of an accretion disc in AG Dra, and conclude that the broad components of the optical emission lines are probably due to the wind of a hot compact object. In addition, Thomson scattering may affect the line wings.

Our observations do not include the largest amplitude outbursts like those in 1980, 1981 and 1994, and we have not detected extremely broad emission wings extending up to 2000-2500 or even $3000 \mathrm{~km} \mathrm{~s}^{-1}$. Our broadest lines extend up to about $1000 \mathrm{~km} \mathrm{~s}^{-1}$ from the line centre. There is certain subjective aspect in drawing the continuum level under the spectral lines, but it is unlikely that this could cause differences in the extent of the wings of more than a few hundred $\mathrm{km} \mathrm{s}^{-1}$. More likely, the hot stellar wind from the white dwarf in AG Dra did not reach its maximum possible velocity during the minor outbursts in 1997 and 1998.

Ionization potentials of the ions generating emission lines in the spectrum of AG Dra cover a wide range, extending from $13.6 \mathrm{eV}$ (H I) through $54.4 \mathrm{eV}$ (He II) to $113.9 \mathrm{eV}$ (O VI). In general, both low- and high-excitation lines behave in a similar way, becoming stronger when the star becomes brighter (Fig. 2). Of special interest is the Raman scattered O VI line 


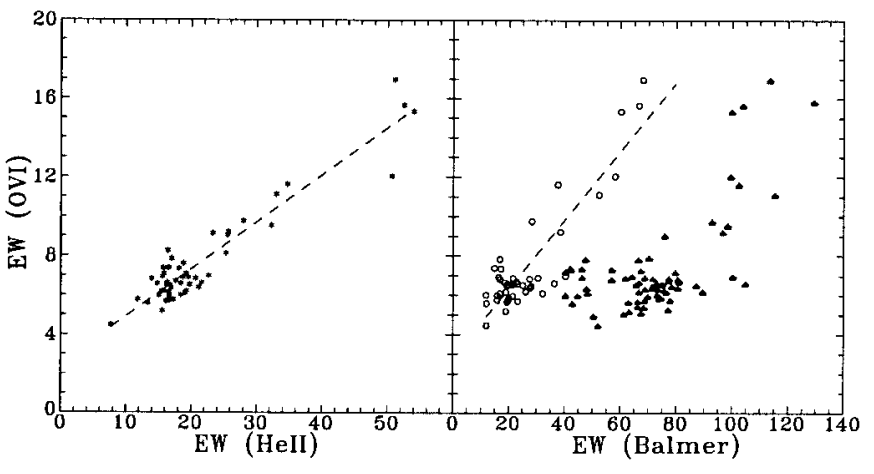

Fig. 8. Left panel: $E W$ of the OVI $\lambda 6825$ realtive to that of the He II $\lambda 4686$ line. Right panel: the same for the hydrogen Balmer lines. Open circles denote $E W \mathrm{~s}$ of the $\mathrm{H} \beta$ line, filled triangles that of $\mathrm{H} \alpha$.

at $\lambda 6825$ (and its counterpart at $\lambda 7082$, not included in the present observations). Those lines, encountered only in symbiotic stars, remained unexplained for a long time until Schmid (1989) proposed that they form when photons of the O VI resonance doublet $\lambda \lambda$ 1032, 1038 undergo Raman scattering off the neutral hydrogen atoms. Up to now, Raman scattered lines are found only in the spectra of symbiotic stars and planetary nebulae (e.g. Arrieta \& Torres-Peimbert 2003). Only about half of all symbiotic stars exhibit $\lambda \lambda$ 6825, 7082 lines (Belczyński et al. 2000).

Are variations in the Raman $\lambda 6825$ line caused by changes in the number of O VI photons, by variations in the amount of neutral hydrogen, or both? The He II $\lambda 4686$ line, most likely, forms close to the hot component, and its intensity, at least to some extent, traces the variations in the number of O VI photons. The hydrogen Balmer lines, in turn, originate from a wider region of the ionized nebula. Figure 8 presents the dependence of the intensity of the Raman scattered OVI $\lambda 6825$ line on that for the $\mathrm{He}$ II $\lambda$ 4686, $\mathrm{H} \alpha$, and $\mathrm{H} \beta$. Although with some scatter, a linear relation $W_{\lambda}(6825)=0.24 W_{\lambda}(\mathrm{He}$ II $)+2.50$ rather well approximates the data points (Fig. 8, left panel). A similar relation $W_{\lambda}(6825)=0.17 W_{\lambda}(\mathrm{H} \beta)+2.84$ can be drawn on the right panel of Fig. 8. However, scatter in the $\mathrm{H} \beta$ data points is much greater than that in the He II points. The rms deviations of the data points from the approximation line are 1.587 and 0.697 , respectively. The values of $W_{\lambda}(\mathrm{H} \beta)$ between 10 and $40 \AA$ may correspond to approximately the same values of $W_{\lambda}(6825)$. There is no correlation between $W_{\lambda}(6825)$ and $W_{\lambda}(\mathrm{H} \alpha)$ up to values of $W_{\lambda}(\mathrm{H} \alpha) \sim 80 \AA$. For higher values, a linear approximation can be drawn, but the scatter is still large.

Correlation between the He II and $\mathrm{O}$ VI lines implies that greater number of $\mathrm{O}$ VI photons might be responsible for the stronger $\lambda 6825$ line. But the increased number of high energy photons would ionize a more extended region of the nebula, therefore reducing the scattering efficiency. The intensity of the $\lambda 6825$ line still increases with increasing $U$ brightness (which, in turn, indicates enhanced nebular emission due to the increasing number of recombining ions), and with correspondingly strengthening Balmer lines. This means that shrinking of the neutral scattering region should be somehow compensated, most naturally by an enhanced stellar wind from the cool giant.
Schmid et al. (1999) have found a very high Raman scattering efficiency in AG Dra compared to other symbiotic stars studied. The efficiency, defined as the photon ratio $N_{\text {Raman }} / N_{\text {OVI }}$ between the Raman scattered and the initial line component, reached $49 \%$ for $N_{6825} / N_{1032}$ both in 1993 and 1996 , and $48 \%$ and $32 \%$, respectively, for $N_{7082} / N_{1038}$. In four other systems those ratios were below $10 \%$, but in the system CD-43 $14304 N_{6825} / N_{1032}$ reached $27 \%$. Interestingly, this is also a symbiotic star with an early type cool component, as are also HK Sco and Hen 3-1342. These systems have rather smooth Raman line profiles with a red peak and a blue shoulder. Symbiotic stars with late M giants or Mira variables tend to show more structured Raman line profiles with two or three intensity peaks (Schmid \& Schild 1997 and references therein). Birriel et al. (2000) found much lower Raman scattering efficiencies in AG Dra in 1995, $14 \%$ and $13 \%$ for $N_{6825} / N_{1032}$ and $N_{7082} / N_{1038}$, respectively. But still, these values are higher than for other stars in their study.

The information given above indicates that cool giant plays a significant role in the activity of the AG Dra system. The stellar wind of the giant likely is modulated by pulsations of the star that take place with the 355-day period, and might be non-radial (Gális et al. 1999). Shells ejected from the giant are photoionized by the hot component, and thus, intensities of the recombination lines also roughly follow this pulsational period, at least in outburst stages.

González-Riestra et al. (1999) have found the ratio of the He II $\lambda 4686$ and $\mathrm{H} \beta$ line fluxes to be on average $0.8 \pm 0.2$. Our data give practically the same value: $0.79 \pm 0.15$. The only episode with $\mathrm{He} \mathrm{II} / \mathrm{H} \beta \geq 1$ was observed around JD 2451800 ... 2451900 (October to December 2000). This coincides with the lowest $\mathrm{H} \alpha$ intensities in our data. Consequently, Balmer lines were unusually weak at this time, while He II $\lambda 4686$ and O VI $\lambda 6825$ did not change much. It is interesting to note that González-Riestra et al. (1999) detected a similar episode, characterictic of hot outbursts, in 1986, about 14-15 years prior to our observations. It would be tempting to say that both major cool outbursts and smaller scale hot outbursts in AG Dra begin with about 15-year intervals, but the present data are too scarce to confirm that. However, according to Mikołajewska (2002) all radio detections of AG Dra prior to 2000 were made during hot outbursts (in 1986). The star was not detected in 1982, in the middle of the large cool outburst (Seaquist \& Taylor 1990) nor during quiescence in 1991 (Seaquist et al. 1993). The next detection by MERLIN in March 2000 (Ogley et al. 2002) is quite close to the time when $\mathrm{He} \mathrm{II} / \mathrm{H} \beta$ was greater than unity. As there are only 6 occasions over 18 years when radio observations of AG Dra have been accomplished, it is not possible to make further conclusions about the relations between radio flux and optical emission lines. However, it is clear that for thermal radio emission to be detectable, quite a large volume of ionized hydrogen must be present in the system. As estimated by Mikołajewska (2002), it is likely that bipolar radio lobes observed in 2000 were ejected during the series of outbursts in 1995-1998. Similarly, the extended radio emission in 1986 (Seaquist \& Taylor 1990; Torbett \& Campbell 1987) may originate from large scale outbursts in early 1980s. 
Bipolar radio emission may imply that it originates in fast collimated jets as in some other symbiotic stars, e.g. MWC 560, CH Cyg, R Aqr, Hen3-1341, RS Oph (Belczyński et al. 2000; Leedjärv 2002 and references therein). Such jets are usually associated with an accretion disc, the presence of which in the AG Dra system is unlikely. Recent hydrodynamical simulations by Gawryszczak et al. $(2002,2003)$ have shown that in S-type symbiotic systems the wind of the red giant is significantly deflected towards the orbital plane by the gravitational pull of the secondary. This results in an enhanced density region in the orbital plane, with a disc-like condensation surrounding the hot component. Such a density distribution significantly affects the size and shape of the $\mathrm{H}$ II region where the radio emission arises. As a result, a bipolar radio map similar to that observed in AG Dra may be obtained without invoking an accretion disc. Enhanced density of neutral material in the orbital plane may also contribute to the origin of strong Raman scattered lines.

The main results of our studies and discussions on AG Dra are as follows:

1. Optical emission lines studied ( $\mathrm{H} \alpha, \mathrm{H} \beta, \mathrm{He}$ II $\lambda$ 4686, Raman scattered OVI $\lambda$ 6825) are variable, with their equivalent widths correlating with the $U$ brightness of the star.

2. There is no apparent correlation of the intensities of the emission lines with the orbital motion (except $\mathrm{H} \beta$ in quiescence, see Fig. 5).

3. The strongest emission lines are observed about $70-175$ days after the maximum pulsational velocity of the cool giant according to the ephemeris of Gális et al. (1999), or 20-95 days after the photometric maximum in the $U$ light according to the ephemeris of Bastian (1998).

4. Our searches have revealed a possible period of about 390 days for the equivalent widths of all the lines studied. Its significance, however, is low, its difference from the period of Bastian (378.5 days) may be due to the small number of data points with high values. Nevertheless, modulated wind from the pulsating giant and possible resonances with the orbital motion seem to be significant factors in for behaviour of AG Dra with about a one year cycle.

5. $\mathrm{H} \alpha$ profiles are always smooth in the outbursts, while during quiescence a weak absorption component on the blue side is observed between orbital phases 0.30 and 0.67 (around photometric minimum).

6. Coincidence of the occasions when AG Dra was detectable at radio wavelengths and when the He II $\lambda 4686$ line was stronger than the $\mathrm{H} \beta$ line (in 1986 and 2000) and the fact that both cases happened during a so called hot outburst may help our understanding the nature of the hot component and its outbursts.

7. Further multiwavelength observations, obtained as frequently as possible are of high importance even for such well studied symbiotic stars as AG Dra.

Acknowledgements. The authors are grateful to master T. Eenmäe and A. Hirv from Tartu University for taking some spectra of AG Dra, and also to several former students from Nicolaus Copernicus and Jagiellonian Universities, especially J. Ł. Janowski and S. M. Frąckowiak for their photometric observations with the $60 \mathrm{~cm}$ reflector in Piwnice. Dr. J. Pelt is acknowledged for advice on the ISDA package. Remarks by the referee, Dr. R. L. M. Corradi, helped to make the paper more concise and legible. The present study was supported by the Estonian Science Foundation grant No. 5003 and by the Polish KBN grant No. 5 P03D 00320.

\section{References}

Arrieta, A., \& Torres-Peimbert, S. 2003, ApJS, 147, 97

Bastian, U. 1998, A\&A, 329, L61

Belczyński, K., Mikołajewska, J., Munari, U., Ivison, R. J., \& Friedjung, M. 2000, A\&AS, 146, 407

Birriel, J. J., Espey, B. R., \& Schulte-Ladbeck, R. E. 2000, ApJ, 545, 1020

Corradi, R. L. M., Mikołajewska, J., \& Mahoney, T. J. (eds.) 2003, Symbiotic Stars Probing Stellar Evolution, ASP Conf. Ser., 303, in press

Fekel, C. F., Hinkle, K. H., Joyce, R. R., \& Skrutskie, M. F. 2000, AJ, 120,3255

Friedjung, M. 1997, in Physical Processes in Symbiotic Binaries and Related Systems, ed. J. Mikołajewska, Warsaw, 105

Friedjung, M., Hric, L., Petrík, K., \& Gális, R. 1998, A\&A, 335, 545

Friedjung, M., Gális, R., Hric, L., \& Petrík, K. 2003, A\&A, 400, 595

Gális, R., Hric, L., Friedjung, M., \& Petrík, K. 1999, A\&A, 348, 533

Gawryszczak, A. J., Mikołajewska, J., \& Różyczka, M. 2002, A\&A, 385,205

Gawryszczak, A. J., Mikołajewska, J., \& Różyczka, M. 2003, A\&A, 398, 159

González-Riestra, R., Viotti, R., Iijima, T., \& Greiner, J. 1999, A\&A, 347,478

Greiner, J., \& DiStefano, R. 2002, A\&A, 387, 944

Greiner, J., Bickert, K., Luthardt, R., et al. 1997, A\&A, 322, 576

Iijima, T., Vittone, A., \& Chochol, D. 1987, A\&A, 178, 203

Kafatos, M., Meier, S. R., \& Martin, I. 1993, ApJS, 84, 201

Kahabka, P. 1998, A\&A, 331, 328

Kaler, J. 1987, AJ, 94, 437

Kenyon, S. J. 1988, AJ, 96, 337

Leedjärv, L. 2002, in The Physics of Cataclysmic Variables and Related Objects, ed. B. T. Gänsicke, K. Beuermann, \& K. Reinsch, ASP Conf. Ser., 261, 353

Meinunger, L. 1979, IBVS, 1611

Mikołajewska, J. 2002, MNRAS, 335, L33

Mikołajewska, J., Kenyon, S. J., Mikołajewski, M., Garcia, M. R., \& Polidan, R. S. 1995, AJ, 109, 1289

Montagni, F., Maesano, M., Viotti, R., et al. 1996, IBVS, 4336

Mürset, U., \& Schmid, H.-M. 1999, A\&AS, 137, 473

Ogley, R. M., Chaty, S., Crocker, M., et al. 2002, MNRAS, 330, 772

Pakull, M. W., Moch, C., Bianchi, L., et al. 1993, A\&A, 278, L39

Petrík, K., Hric, L., Gális, R., Friedjung, M., \& Dobrotka, A. 1998, IBVS, 4588

Reinsch, K., van Teeseling, A., Beuermann, K., \& Abbott, T. M. C. 1996, A\&A, 309, L11

Reinsch, K., van Teeseling, A., King, A. R., \& Beuermann, K. 2000, A\&A, 354, L37

Robinson, K., Bode, M. F., Skopal, A., Ivison, R. J., \& Meaburn, J. 1994, MNRAS, 269, 1

Schmid, H.-M. 1989, A\&A, 211, L31

Schmid, H.-M., \& Nussbaumer, H. 1993, A\&A, 268, 159

Schmid, H.-M., \& Schild, H. 1997, A\&A, 321, 791

Schmid, H.-M., Krautter, J., Appenzeller, I., et al. 1999, A\&A, 348, 950

Seaquist, E. R., \& Taylor, A. R. 1990, ApJ, 349, 313

Seaquist, E. R., Krogulec, M., \& Taylor, A. R. 1993, ApJ, 410, 260 
Skopal, A. 1998, Contr. Skaln. Pl., 28, 87

Skopal, A., Vaňko, M., Pribulla, T., et al. 2002, Contr. Skaln. Pl., 32, 62

Smith, S. E., \& Bopp, B. W. 1981, MNRAS, 195, 733

Smith, V. V., Cunha, K., Jorissen, A., \& Boffin, H. M. J. 1996, A\&A, 315,179

Sokoloski, J. L., Bildsten, L. \& Ho, W. C. G. 2001, MNRAS, 326, 553

Southwell, K., Livio, M., Charles, P. A., O’Donoghue, D., \& Sutherland, W. J. 1996, ApJ, 470, 1065
Tomov, N. A., \& Tomova, M. T. 2000, IBVS, 4953

Tomov, N. A., \& Tomova, M. T. 2002, A\&A, 388, 202

Tomova, M. T., \& Tomov, N. A. 1998, IBVS, 4561

Tomova, M. T., \& Tomov, N. A. 1999, A\&A, 347, 151

Torbett, M. V., \& Campbell, B. 1987, ApJ, 318, L29

Viotti, R., Ricciardi, O., Ponz, D., et al. 1983, A\&A, 119, 285

Viotti, R., Rossi, C., \& Muratorio, G. 1998, A\&AS, 128, 447

Zhu Z. X., Friedjung, M., Zhao, G., Hang, H. R., \& Huang, C. C. 1999, A\&AS, 140, 69 